\title{
Charm and Beauty Production from Semileptonic Decays at HERA
}

\author{
Markus Jüngst* \\ Universität Bonn \\ E-mail: juengst@physik.uni-bonn.de
}

Charm and beauty production are key components of the HERA physics programme. I will summarise a selection of the recent results obtained by the ZEUS collaboration. One possibility to tag heavy flavour events is the identification of leptons from the semileptonic decay. The production of beauty in deep inelastic scattering will be discussed for final states using muons or electrons from the decay. In one measurement using muons, it was also possible to measure the charm contribution to the full cross section. The extracted beauty contribution to the proton structure function, $F_{2}^{b \bar{b}}$, is compared with other results which use independent techniques.

35th International Conference of High Energy Physics

July 22-28, 2010

Paris, France

${ }^{*}$ for the ZEUS collaboration 


\section{Introduction}

Heavy flavour production in $e^{ \pm} p$ collisions at HERA provides a good testing ground of perturbative Quantum Chromodynamics (pQCD) as the high quark mass provides a hard scale. Furthermore, the existence of other hard scales such as $Q^{2}$, the virtuality of the exchanged boson, or $p_{t}$, the transverse momentum of the heavy quark, allow resummation techniques to be tested. Different kinematic variables are used to describe the $e p$ interaction at HERA: $Q^{2}$, the Bjorken scaling variable, $x$, and the inelasticity, $y$. Until 1997 HERA ran at a centre-of-mass energy of $\sqrt{s}=300 \mathrm{GeV}$. This energy was increased to $\sqrt{s}=320 \mathrm{GeV}$ for data taken from 1998 onwards. Due to improvements of the detector and accelerator during a break in the data taking, the available dataset is split into two periods. From 1996 till 2000 in the HERA I period about $130 \mathrm{pb}^{-1}$ and between 2003 and 2007 (HERA II) about $400 \mathrm{pb}^{-1}$ per experiment were collected.

The kinematic range of the analysed data can be separated into the following two regimes: photoproduction $(\gamma p)$ where the exchanged photon in the process is almost real; and deep inelastic scattering (DIS) where the exchanged photon is virtual. Experimentally, $\gamma p$ is defined by the scattered electron not being in the acceptance region of the detector, corresponding to a cut $Q^{2} \lesssim 1 \mathrm{GeV}^{2}$.

\section{Theory}

To evaluate the detector acceptance and provide signal and background distributions, samples generated with the RAPGAP[1] Monte Carlo program were used. In all three analyses the measured cross sections are also compared with next-to-leading order (NLO) QCD prediction obtained in the fixed-flavour-number scheme (FFNS) using the HVQDIS program. The extracted proton structure functions $F_{2}^{b \bar{b}}$ are compared with predictions by the CTEQ[2] and MSTW[3] groups using NLO calculations based on the general-mass variable number scheme (VFNS) with different treatments of the flavour-threshold. The prediction of GJR[4] is based on the FFNS whereas the prediction of $\mathrm{ABKM}[5]$ is based on the partial NNLO FFNS calculation.

\section{Semileptonic decays into muons}

In this analysis, which uses the full HERA I dataset, the kinematic properties of the muon from semileptonic $b$ decays was used to determine beauty cross sections over a wide range of $Q^{2}$. Using the different shapes of $p_{T}^{\text {rel }}$, the transverse momentum of the muon relative to the associated jet axis, it was possible to determine cross sections for $Q^{2}>2 \mathrm{GeV}^{2}$. Figure 1 shows the differential cross section as a function of $Q^{2}$. The shape of the distribution is reproduced by both the RAPGAP leading order Monte Carlo and the NLO

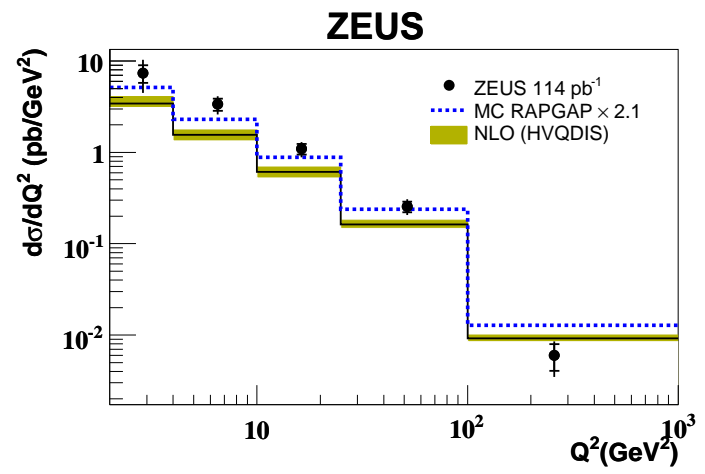

Figure 1: Differential cross section as a function of $Q^{2}$.

QCD prediction, where both predictions underestimate the data. In addition the cross sections were also determined as a function of $p_{T}^{\mu}, \eta^{\mu}, p_{T}^{j e t}$ and $\eta^{j e t}$, and, in order to extract $F_{2}^{b \bar{b}}$, as a function of $Q^{2}$ and $x$. 


\section{ZEUS}
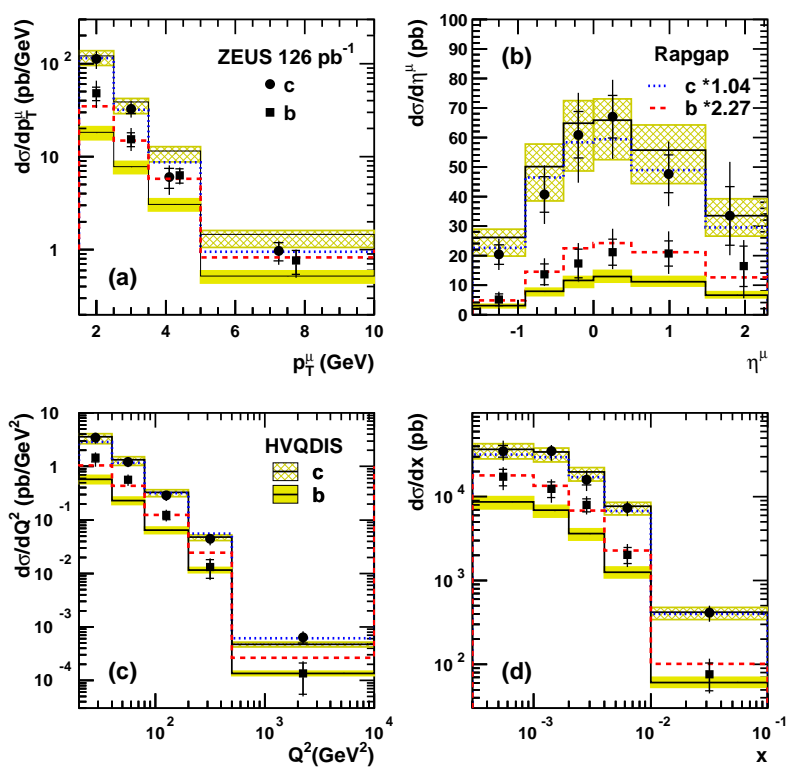

Figure 2: Differential charm and beauty cross sections as a function of $p_{T}^{\mu}, \eta^{\mu}, Q^{2}$ and $x$.

In the second muon analysis, based on the 2005 running period, the sensitivity was increased by using two additional variables in the fit. The charm detection sensitivity was increased by adding $p_{T}^{\text {miss } \| \mu}$, the component of the missing momentum vector parallel to the muon. In addition the beauty and charm separation was improved by using $\delta$, the distance of closest approach of the muon to the interaction region. Figure 2 shows the differential charm and beauty cross sections as a function of $p_{T}^{\mu}, \eta^{\mu}, Q^{2}$ and $x$. The NLO QCD calculation is in good agreement for charm while the beauty cross section is 2.3 standard deviations above the HVQDIS result. $F_{2}^{c \bar{c}}$ and $F_{2}^{b \bar{b}}$ were extracted from double differential cross sections in the region of $Q^{2}>20 \mathrm{GeV}^{2}$.

\section{Semileptonic decays into electrons}

In this analysis based on the full HERA II dataset the electron decay channel was investigated, where it was possible to lower the cut on the lepton momentum. Instead of a simultaneous fit of several variables, they were combined into one discriminating variable. Figure 3 shows the fit of the likelihood test function, which combines variables sensitive to the electron identification with variables sensitive to the decay channel. From the fitted contribution of electrons from semileptonic beauty decays, other electrons and background particles, the beauty cross sections as

ZEUS

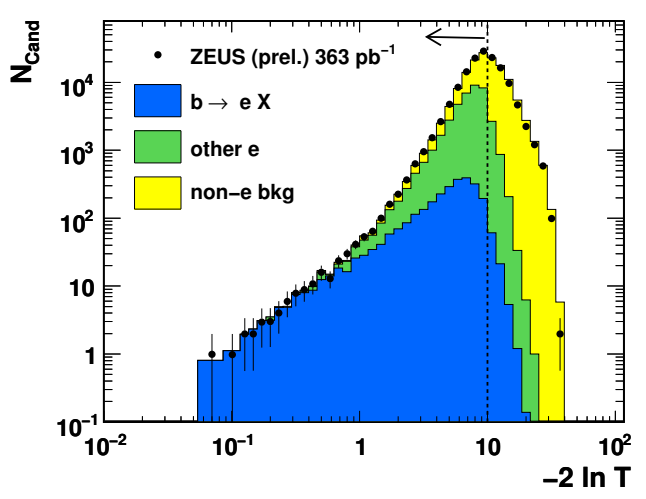

Figure 3: Test function distribution. a function of $p_{T}^{e}, \eta^{e}$ (see Figure 4), $Q^{2}$ and $x$ were extracted.

The scaled RAPGAP prediction and the NLO QCD calculation are in good agreement with the measured values. $F_{2}^{b \bar{b}}$, which is shown in the next section, was extracted for $Q^{2}$ above $10 \mathrm{GeV}$. 


\section{ZEUS}
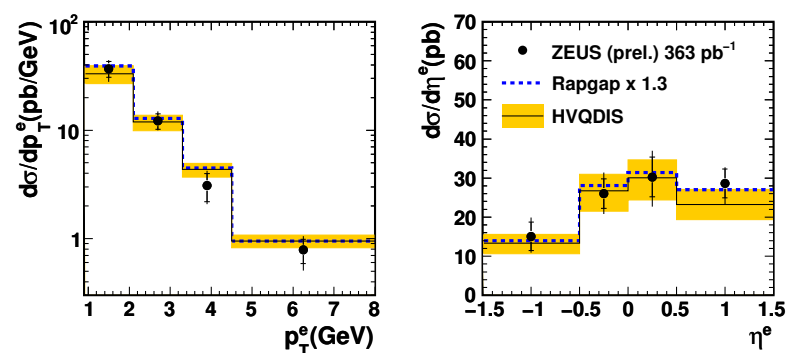

Figure 4: Differential beauty cross section as a function of $p_{T}^{e}$ and $\eta^{e}$.

\section{Conclusion}

In the presented analyses the beauty contribution to the proton structure function, $F_{2}^{b \bar{b}}$ was extracted from double differential cross sections as a function of $Q^{2}$ and $x$. Figure 5 shows the resulting measurements of $F_{2}^{b \bar{b}}$. In addition to the analyses using semileptonic decays, also two inclusive measurements from H1[9] and ZEUS[10] are shown. The extracted points are compared to NLO and NNLO QCD predictions using different theoretical approaches for the calculations. The prediction using the HVQDIS program is shown with its uncertainty indicated by the band. All measurements are consistent with each other and agree well with the theoretical prediction.

Using semileptonic decays to muons or electrons, beauty production in DIS was measured over a wide range in $x$ and $Q^{2}$ and gives a consistent picture. In comparison to the first analyses, later measurements benefited from the addi-

tional information of the microvertex detector in the HERA II dataset. The lifetime information improved the sensitivity to the identification of heavy flavour events, especially for beauty production. In one of the presented analyses using the decay into muons it was also possible to extract the charm contribution to the proton structure function, $F_{2}^{c \bar{c}}$.

\section{HERA}

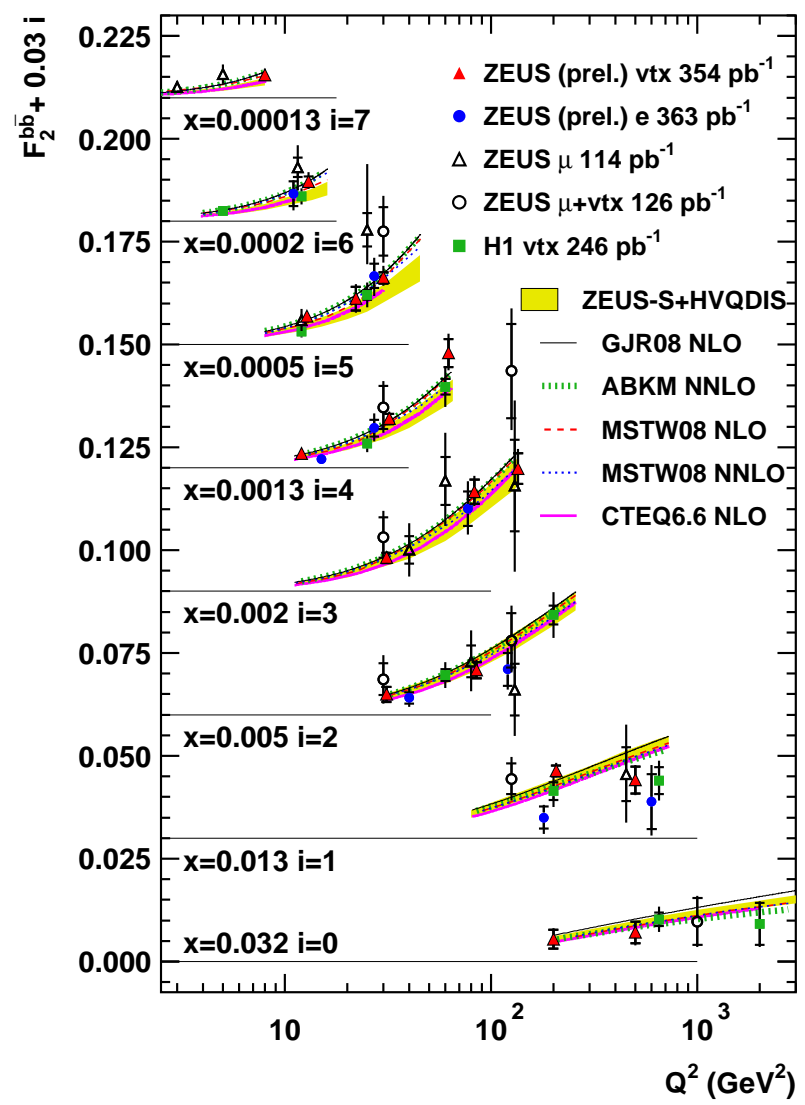

Figure 5: Measurement of $F_{2}^{b \bar{b}}$. 


\section{References}

[1] H. Jung, Comp. Phys. Comm 86, 147 (1995)

[2] CTEQ Coll., P.M. Nadolsky et al., Phys. Rev. D 78, 013004 (2008)

[3] A.D. Martin et al., Eur. Phys. J. C 63, 189 (2009)

[4] M. Glück, P. Jiminez-Delgado and E. Reya, Eur. Phys. J. C 53, 355 (2008)

[5] S. Alekhin and S. Moch, Phys. Lett. B 672, 166 (2009)

[6] ZEUS Coll., H. Abramowicz et al. , Measurement of beauty production in DIS and $F_{2}^{b \bar{b}}$ extraction at ZEUS, DESY-10-47 (April 2010)

[7] ZEUS Coll., S. Chekanov et al., Eur. Phys. J. C 65, 65 (2010)

[8] ZEUS Coll., H. Abramowicz et al. , Beauty production in DIS using decays into electrons at HERA, ZEUS-prel-10-010 (July 2010)

[9] H1 Coll., F. .D. Aaron et al., Eur. Phys. J. C 65, 89 (2010)

[10] ZEUS Coll., H. Abramowicz et al. , Beauty from inclusive secondary vertices in DIS, ZEUS-prel-10-004 (July 2010) 